\title{
A GENERALIZATION OF THE BANACH AND MAZUR GAME
}

\author{
BY \\ HAIM HANANI
}

1. Introduction. 11. The definition of the game. Given a sequence $0<k_{1}$ $\leqq k_{2} \leqq \cdots \leqq k_{m} \leqq \cdots$ we define a game $G\left\{k_{m}\right\}$ as follows: Two players $A$ and $B$ choose alternately positive numbers $x_{n},(n=0,1,2, \cdots)$ according to the following rules: $B$ starts by choosing $x_{0}>0$; after $x_{i},(i=0,1, \cdots, 2 n-2)$ have been chosen, $A$ chooses $x_{2 n-1}$ such that

$$
0<x_{2 n-1}<x_{2 n-2}
$$

and subsequently $B$ chooses $x_{2 n}$ such that

$$
0<x_{2 n}<k_{n} x_{2 n-1} \text {. }
$$

Given a set $S \subset[0, \infty), A$ will be said to win on $S$ if $\sum_{i=0}^{\infty} x_{i}=s \in S$; otherwise, $B$ wins.

We say that the set $S$ is unavoidable ( $B$ cannot avoid $S$ ), if $A$ has a winning strategy on $S$, i.e. if there exists a sequence of functions

$$
x_{2 n+1}\left(x_{0}, x_{1}, \cdots, x_{2 n}\right), \quad(n=0,1,2, \cdots)
$$

satisfying (1.1) and such that $s=\sum_{i=0}^{\infty} x_{i} \in S$ whenever $x_{2 n},(n=0,1,2, \cdots)$ satisfy (1.2). If, on the other hand, $B$ has a winning strategy on $S$, we say that $S$ is avoidable ( $B$ can avoid $S$ ).

In the sequel we shall also consider a game $\widetilde{G}\left\{k_{m}\right\}$, defined as a game $G\left\{k_{m}\right\}$ satisfying the additional condition $x_{0}<1$.

$\tilde{G}\left\{k_{m}\right\}$ will be played exclusively on bounded sets $S_{0} \subset[0,1]$.

12. Historical notes. Various variants of the game of Banach and Mazur are described in the so-called Scottish Book (see Colloq. Math. vol. 1 (1947) p. 57). One of them, which was defined by $S$. Mazur and later modified by $\mathrm{S}$. Banach, is a special case of our game for $k_{m}=1,(m=1,2, \cdots)$. This case was first considered by A. Turowicz [4], who proved that the set of all irrational numbers is unavoidable. His result was later generalized by S. Zubrzycki [5], who has shown that the complement of any countable set is unavoidable. A sufficient condition for avoidability of sets was given by S. Hartman [2]. Further, M. Reichbach [3] has constructed (for $k_{m}=1$ ) a perfect unavoidable set of measure 0 and thus has given an answer to a question put by $\mathrm{H}$. Steinhaus, concerning the existence of unavoidable sets of the first category.

Received by the editors December 12, 1958. 
S. Banach posed the still unsolved question, what are the necessary and sufficient conditions for an arbitrary set $S$ to be unavoidable (in the case $\left.k_{m}=1\right)$. Some contributions to the solution of this problem and a full characterization of a class of unavoidable sets will be given in [1].

13. Outline of results. In this paper (with the exception of the last section) we shall confine ourselves to closed sets only.

It will be shown (Theorem 1) that for every game $G\left\{k_{m}\right\}$ there exists an unavoidable set $S$ which is nowhere dense and perfect.

As regards the measure of unavoidable sets, we shall prove (Theorem 2) that a necessary and sufficient condition for the existence of a perfect (or closed) unavoidable set of measure 0 is $\sum_{m=1}^{\infty} 1 / k_{m}=\infty$. For the game $\tilde{G}\left\{k_{m}\right\}$ it will be moreover proved (Theorem 3 ) that the infimum of measures of all the perfect (or closed) unavoidable subsets of $[0,1]$ is

$$
\prod_{m=1}^{\infty}\left(1-1 /\left(k_{m}+1\right)\right) \text {. }
$$

Universal unavoidable sets $U U S G$ and $U U S \tilde{G}$ are sets which are unavoidable for every game $G\left\{k_{m}\right\}$ or $\tilde{G}\left\{k_{m}\right\}$ respectively. It will be shown (Theorems 4 and 5) that a $U U S G$ is of the second category in every point of $[0,1]$, and a $U U S G$ is of the second category in every point of $[M, \infty)$ for some $M$. Finally we shall construct non-trivial examples of a $U U S G$ and a UUSĞ.

14. Notation. Let $a$ be any interval with endpoints $x, y,(x \leqq y)$; we denote:

$$
\begin{aligned}
\bar{a} & =[x, y]=\{z: x \leqq z \leqq y\}, \\
a^{\circ} & =(x, y)=\{z: x<z<y\}, \\
{ }^{\prime} a & =[x, y)=\{z: x \leqq z<y\},
\end{aligned}
$$

$l(a)=x$, the left endpoint of $a$,

$r(a)=y$, the right endpoint of $a$,

$|a|=y-x$ is the length of interval $a$, we use also $a$ for $|a|$ when meaning is clear by context; thus e.g. $U a_{i}$ will denote the union of intervals, but $\sum a_{i}$ the sum of their lengths.

By $f$ we shall denote as a rule closed intervals $(f=\bar{f})$; by $g$, open ones $\left(g=g^{\circ}\right)$.

Let $S$ be any set $S \subset[0, \infty)$; we denote: $m(S)$, the Lebesgue measure of $S$; $S+t=\{x+t: x \in S\}$, the translate of $S$ by $t$, ( $t$ is a number); $\bar{S}$, the closure of $S ; C(S)=[0, \infty) \sim S$, the complement of $S$ with regard to $[0, \infty)$, for $S_{0} \subset[0,1] ; C\left(S_{0}\right)=[0,1] \sim S_{0}$, the complement of $S_{0}$ with regard to $[0,1]$.

Further we shall denote $s_{n}=\sum_{i=0}^{n} x_{i}$. Evidently the sequence $s_{n}$, $(n=0,1,2, \ldots)$ is monotonically increasing, and if it converges then $\lim _{n \rightarrow \infty} s_{n}=\sum_{i=0}^{\infty} x_{i}=s$. 
2. The existence of unavoidable nowhere-dense sets.

THEOREM 1. For every game $G\left\{k_{m}\right\}$ there exists an unavoidable set $S$ which is nowhere dense and perfect.

Proof. We begin by proving the theorem for $\widetilde{G}\left\{k_{m}\right\}$. We shall namely construct a perfect nowhere dense set $S_{0} \subset[0,1]$, which will turn out to be unavoidable.

Let $f=[0,1]$, and decompose $f$ into 3 subintervals

$$
f_{0}=\left[0, \frac{k_{1}+1}{2 k_{1}+3}\right], \quad g=\left(\frac{k_{1}+1}{2 k_{1}+3}, \frac{k_{1}+2}{2 k_{1}+3}\right), \quad f_{1}=\left[\frac{k_{1}+2}{2 k_{1}+3}, 1\right] \text {. }
$$

Similarly decompose for every $n,(n=1,2, \cdots)$ each of the closed intervals $f_{\delta_{1}} \cdots \delta_{n-1},\left(\delta_{i}=0,1 ; i=1,2, \cdots, n-1\right)$ into

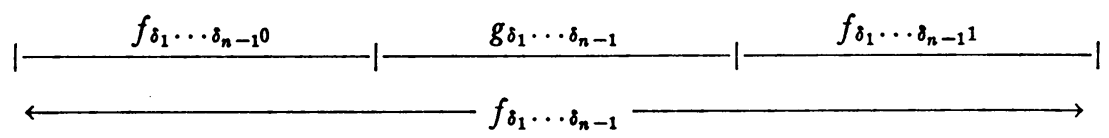

2 closed subintervals $f_{\delta_{1}} \cdots \delta_{n-1} 0$ and $f_{\delta_{1}} \cdots \delta_{n-1}{ }^{1}$ and an open one $g_{\delta_{1}} \cdots \delta_{n-1}$ so that

$$
\begin{aligned}
& \left|f_{\delta_{1}} \ldots \delta_{n}\right|=\frac{k_{n}+n}{2 k_{n}+2 n+1}\left|f_{\delta_{1} \cdots \delta_{n-1}}\right|, \quad\left(\delta_{n}=0,1\right) \\
& \left|g_{\delta_{1} \cdots \delta_{n-1}}\right|=\frac{1}{2 k_{n}+2 n+1}\left|f_{\delta_{1} \cdots \delta_{n-1}}\right| \text {. }
\end{aligned}
$$

Let now $S_{0}=\bigcap_{n-0}^{\infty} U_{\delta_{i}-0,1 ; i-1,2}, \ldots, n f_{\delta_{1}} \ldots \delta_{n}$. Evidently $S_{0}$ is homeomorphic with the Cantor set and as such is perfect and nowhere dense.

We shall now prove that $A$ has a winning strategy on $S_{0}$.

$A$ is said to be in a winning position of the first kind after his $n$th move $x_{2 n-1}, A \in W_{n}$, if for some $g_{\delta_{1}} \ldots \delta_{m-1},(m \geqq n)$

$$
s_{2 n-1}=r\left(g_{\delta_{1}} \ldots \delta_{m-1}\right)
$$

and

$$
k_{n} x_{2 n-1} \leqq\left|f_{\delta_{1}} \ldots \delta_{m}\right|,
$$$$
\left(\delta_{m}=1\right) .
$$

Similarly $A$ will be said to be in a winning position of the second kind after his $n$th move, $A \in W_{n}^{\prime}$, if for some $g_{\delta_{1}} \cdots_{\delta_{m} e_{1}} \cdots e_{\mu},(m \geqq n, \mu \geqq 0)$

$\left(\alpha_{n}^{\prime}\right)$

$\left(\beta_{n}^{\prime}\right)$

$$
\begin{aligned}
s_{2 n-1} & =r\left(g_{\delta_{1}} \cdots \delta_{m_{1}} \cdots e_{\mu}\right), \\
k_{n} x_{2 n-1} & \leqq r\left(f_{\delta_{1}} \cdots \delta_{m}\right)-s_{2 n-1}
\end{aligned}
$$

and for every $\nu$ satisfying $0 \leqq \nu<\mu$ and $\epsilon_{\nu+1}=0$,

$$
l\left(g_{\delta_{1}} \ldots \delta_{m} e_{1} \ldots \epsilon_{\nu}\right)-s_{2 n-1}>\left|g_{\delta_{1}} \ldots \delta_{m} e_{1} \cdots e_{\nu}\right| .
$$

We shall prove that $A$ has a strategy which enables him to be in one of 
the winning positions after each of his moves. From this follows $s_{2 n-1} \in S_{0}$, $(n=1,2, \cdots)$ and $-S_{0}$ being closed and bounded-also $\lim _{n \rightarrow \infty} s_{2 n-1}$ $=\lim _{i \rightarrow \infty} s_{i}=s \in S_{0}$, i.e. $S_{0}$ is unavoidable.

The proof that $A$ has the mentioned strategy will be given by induction. Agreeing on

$$
s_{-1}=0, k_{0} x_{-1}=|f|=1,
$$

evidently $A \in W_{0}$ at the beginning of the game.

Suppose now that $A \in W_{n}$ or $A \in W_{n}^{\prime}$. It is evident that whatever $x_{2 n}$ is chosen by $B$-provided that (1.2) is satisfied- $s_{2 n} \in f_{\delta_{1}}^{0} \ldots \delta_{m}$ holds. If now for some $g_{\delta_{1}} \cdots \delta_{\lambda},(\lambda \geqq m), s_{2 n} \in^{\prime} g_{\delta_{1}} \cdots \delta_{\lambda}$, then $A$ makes $s_{2 n+1}=r\left(g_{\delta_{1}} \cdots \delta_{\lambda}\right)$. If $A \in W_{n}$, $x_{2 n+1}$ satisfies (1.1) because considering (2.1), $x_{2 n+1} \leqq\left|g_{\delta_{1}} \ldots \delta_{\lambda}\right|<\left|f_{\delta_{1}} \ldots \delta_{\lambda} 0\right|$ $\leqq x_{2 n}$. The same reasoning holds if $A \in W_{n}^{\prime}$ and if $\lambda>m+\mu$, or $\lambda \leqq m+\mu$ and the sequences $\left\{\delta_{m+1}, \delta_{m+2}, \cdots, \delta_{\lambda}\right\},\left\{\epsilon_{1}, \epsilon_{2}, \cdots, \epsilon_{\lambda-m}\right\}$ are not identical (note that in this case if $i$ is the smallest integer such that $\delta_{m+i} \neq \epsilon_{i}$, then necessarily $\epsilon_{i}=0$ and $\left.\delta_{m+i}=1\right)$. If however $\left\{\delta_{m+1}, \cdots, \delta_{\lambda}\right\} \equiv\left\{\epsilon_{1}, \cdots, \epsilon_{\lambda-m}\right\},(\lambda<m+\mu)$ then $x_{2 n+1}<x_{2 n}$ is expressly stipulated by $\left(\gamma_{n}^{\prime}\right)$, as evidently $\epsilon_{\lambda-m+1}=0$. Now after this move $A \in W_{n+1}$. It is namely evident that $\left(\alpha_{n+1}\right)$ is satisfied and considering (2.1), $\left(\beta_{n+1}\right)$ holds as well.

The only other possibility is that

$$
s_{2 n} \in S_{0} \cap f_{\delta_{1}} \cdots \delta_{m}
$$

and $s_{2 n}$ is not a left endpoint of any open interval of $C\left(S_{0}\right)$. Accordingly

$$
s_{2 n}=f_{\delta_{1} \delta_{2}} \ldots \text { def } \bigcap_{r=m}^{\infty} f_{\delta_{1} \delta_{2} \ldots \delta_{r}}
$$

and there are infinitely many zeros among the subscripts of $f_{\delta_{1} \delta_{2}} \ldots$ Evidently

$$
\vartheta_{\tau} \operatorname{def} r\left(f_{\delta_{1}} \ldots \delta_{\tau}\right)-s_{2 n}>0, \quad(\tau=1,2, \cdots)
$$

and

$$
\vartheta_{\tau} \rightarrow 0 \text { monotonically. }
$$

There exists therefore an integer $j$ satisfying

$$
\vartheta_{j}<x_{2 n},
$$$$
(j \geqq m) \text {. }
$$

Denote by $i$ the smallest integer $i>j$ such that $\delta_{i}=0$, and let $p$ be the smallest integer satisfying

$$
\frac{1}{2^{p}}<\frac{1}{k_{n+1}}-\frac{1}{k_{i}+i}
$$

If among the subscripts $\delta_{i+1}, \delta_{i+2}, \cdots$ of $f_{\delta_{1} \delta_{2}} \ldots$ there occurs a sequence of at least $p$ consecutive 1 's, i.e. if there exists an integer $t$ such that 


$$
\delta_{t}=0, \delta_{t+1}=\delta_{t+2}=\cdots=\delta_{t+p}=1 \text {, }
$$

then $A$ makes $s_{2 n+1}=r\left(g_{\delta_{1}} \cdots \delta_{t-1}\right)$. This move of $A$ satisfies (1.1) because $t-1 \geqq j$ and therefore, considering (2.5), (2.6) and (2.7),

$$
x_{2 n+1}=r\left(g_{\delta_{1}} \ldots \delta_{t-1}\right)-s_{2 n}<\vartheta_{j}<x_{2 n} .
$$

Also $A \in W_{n+1}$. It is namely evident that $\left(\alpha_{n+1}\right)$ is satisfied. Moreover, considering (2.10), (2.3) and (2.1), $r\left(f_{\delta_{1}} \ldots \delta_{t+p}\right)=l\left(g_{\delta_{1}} \ldots \delta_{t-1}\right)$ implies

$$
x_{2 n+1} \leqq\left|f_{\delta_{1} \ldots \delta_{t+p}}\right|+\left|g_{\delta_{1} \ldots \delta_{t-1}}\right|<\left(\frac{1}{2^{p}}+\frac{1}{k_{t}+t}\right) \cdot\left|f_{\delta_{1} \cdots \delta_{t}}\right| ;
$$

as $t \geqq i,(2.8)$ gives $1 / 2^{p}+1 /\left(k_{t}+t\right)<1 / k_{n+1}$ and $\left(\beta_{n+1}\right)$ follows.

If on the other hand no sequence (2.9) exists among the subscripts of $f_{\delta_{1} \delta_{2}} \ldots$, denote by $h$ the smallest integer satisfying

$$
k_{h}+h>3^{p}
$$

and let

$$
h<i_{1}<i_{2}<\ldots
$$

be the sequence of all the subscripts $i_{\eta}>h$ satisfying

$$
\delta_{i_{\eta}}=0 \text {, }
$$$$
(\eta=1,2, \cdots)
$$

evidently

$$
i_{\eta+1}-i_{\eta} \leqq p,
$$$$
(\eta=1,2, \cdots)
$$

From (2.4) and (2.13) we have

$$
d_{\eta} \operatorname{def} r\left(g_{\delta_{1}} \ldots \delta_{i_{\eta}-1}\right)-s_{2 n}>0, \quad(\eta=1,2, \cdots)
$$

and $d_{\eta} \rightarrow 0$ monotonically. There exists therefore an integer $\xi$ such that

$$
d_{\xi}<\frac{1}{k_{n+1}+1} \vartheta_{h}
$$

Now $A$ chooses

$$
x_{2 n+1}=d_{\xi}
$$

and makes thus

$$
s_{2 n+1}=r\left(g_{\delta_{1} \cdots \delta_{i \eta-1}}\right) .
$$

Considering (2.15), (2.7) and (2.6) we have, in view of $h>j, d_{\xi}<x_{2 n}$ and therefore by (2.16) $x_{2 n+1}$ satisfies (1.1). Also $A \in W_{n+1}^{\prime}:\left(\alpha_{n+1}^{\prime}\right)$ is namely equivalent to (2.17), $\left(\beta_{n+1}^{\prime}\right)$ follows from (2.16), (2.15) and (2.5); at last by (2.17), (2.13), (2.1), (2.11) and (2.14) 


$$
\begin{aligned}
& l\left(g_{\delta_{1} \cdots \delta_{i_{\eta}-1}}\right)-s_{2 n+1} \geqq l\left(g_{\delta_{1}} \ldots \delta_{i_{\eta}-1}\right)-r\left(g_{\delta_{1} \cdots \delta_{i_{\eta+1}-1}}\right)=\left|f_{\delta_{1} \cdots \delta_{i_{\eta+1}-1,1}}\right| \\
& =\left|g_{\delta_{1} \cdots \delta_{i_{\eta}-1}}\right| \cdot\left(k_{i_{\eta}}+i_{\eta}\right) \cdot \prod_{\lambda=i_{\eta}+1}^{i_{\eta+1}} \frac{k_{\lambda}+\lambda}{2 k_{\lambda}+2 \lambda+1}>\left|g_{\delta_{1} \cdots \delta_{i_{\eta}-1}}\right|, \\
& \quad(\eta=1,2, \cdots, \xi-1)
\end{aligned}
$$

and considering (2.13), $\left(\gamma_{n+1}^{\prime}\right)$ is satisfied.

This accomplishes the proof of the theorem for $\tilde{G}\left\{k_{m}\right\}$ and it remains to extend the proof for $G\left\{k_{m}\right\}$. To this end put $S_{q}=S_{0}+q,(q=1,2, \cdots)$ and

$$
S=\bigcup_{q=0}^{\infty} S_{q} \text {. }
$$

If now $q \leqq x_{0}<q+1$ we fix the strategy of $A$ on $S_{q}$ as described above for $S_{0}$. Consequently $S$ is unavoidable.

REMARK 1. In Theorem 1 the monotonity of the sequence $\left\{k_{m}\right\}$ is not essential. Let $k_{i}^{\prime},(i=1,2, \cdots)$ be any sequence of positive numbers, then the sequence $k_{m}=\max _{1 \leq i \leq m} k_{i}^{\prime},(m=1,2, \cdots)$ is evidently nondecreasing and according to Theorem 1 there exists for $G\left\{k_{m}\right\}$ an unavoidable set $S$ which is nowhere dense and perfect. It is however evident that $S$ is also unavoidable for $G\left\{k_{i}^{\prime}\right\}$.

REMARK 2. Theorem 1 remains evidently true for games $\tilde{G}\left\{k_{m}\right\}$ and bounded sets $S_{0} \subset[0,1]$.

3. The measure of unavoidable sets.

Leмma. For every game $\tilde{G}\left\{k_{m}\right\}$ and every closed unavoidable set $S_{0} \subset[0,1]$, the relation

$$
m\left(S_{0}\right) \geqq \prod_{\mu=1}^{\infty}\left(1-\frac{1}{k_{\mu}+1}\right)
$$

holds.

Proof. Let $S_{0} \subset[0,1]$ be closed. We shall fix such a strategy for $B$ that if $S_{0}$ is unavoidable, then (3.1) is fulfilled.

Clearly 0 and 1 must be nonisolated points of $S_{0}$, as otherwise $B$ could choose $x_{2 j},(j=0,1,2, \cdots)$ so small, or $x_{0}$ so large respectively, as to have a winning strategy. Consequently $C\left(S_{0}\right)$ is a union of at most denumerably many mutually disjoint open intervals, and 0 and 1 are not endpoints of any of them. Let $g_{i},(i=1,2, \cdots, m)$ be any finite set of open intervals of $C\left(S_{0}\right)$ taken in the natural order. In order to prove (3.1) it will evidently suffice to show that

$$
\sum_{i=1}^{m} g_{i}<1-\prod_{\mu=1}^{\infty} \eta_{\mu}
$$

where 


$$
\eta_{\mu}=1-\frac{1}{k_{\mu}+1}, \quad(\mu=1,2, \cdots) .
$$

Denote by $f_{i},(i=1,2, \cdots, m+1)$ the closed intervals of $C\left(\cup_{j=1}^{m} g_{j}\right)$ taken again in the natural order. Some of the intervals $f_{i}$-except of $f_{1}$ and $f_{m+1}$ may shrink to a point. $A$ should never allow

$$
s_{2 n-1} \in{ }^{\prime} g_{i},(n=1,2, \cdots ; i=1,2, \cdots, m)
$$

because in such a case $B$ has a winning strategy, by choosing $x_{2 j}$, $(j=n, n+1, \cdots)$ sufficiently small. (Especially $s_{2 n-1} \in f_{i}$ should not be allowed for $f_{i}$ shrinking to a point.)

In order to fix the strategy of $B$ we shall have to make use of several sequences of moves to be made by $A$ and $B$. The moves of the $\mu$ th sequence will be denoted by $x_{2 \rho_{\mu}+\beta}^{\mu},\left(\beta=0,1,2, \cdots ; \mu=1,2, \cdots ; \rho_{1}=0\right) . B$ begins with $x_{0}^{1}=s_{0}^{1}=r\left(f_{1}\right)$, then $A$ has to make $s_{1}^{1} \in^{\prime} f_{i_{1}}^{1},\left(i_{1}^{1}>1\right)$. If $r\left(f_{i_{1}}^{1}\right)-s_{1}^{1}<k_{1} x_{1}^{1}$, $B$ makes $s_{2}^{1}=r\left(f_{i_{1}}^{1}\right)$. Otherwise $B$ turns to a new sequence of moves and chooses $x_{0}^{2}=s_{0}^{2}=r\left(f_{i_{1}}^{1}\right)$. Generally let $s_{2 \rho_{\mu}+2 \nu}^{\mu}=r\left(f_{i_{\mu}+\nu}\right),(\nu>0)$, then $A$ has to make

$$
s_{2 \rho \mu+2 v+1}^{\mu} \in f_{i_{\mu}+\nu+1}^{\mu},\left(i_{\rho \mu+v+1}^{\mu}>i_{\rho \mu+v}^{\mu}\right)
$$

If

$$
r\left(f_{i_{\mu}^{\mu}+\nu+1}^{\mu}\right)-s_{2 \rho \mu+2 v+1}^{\mu}<k_{\rho_{\mu+\nu+1}}^{\mu} x_{2 \rho \mu+2 v+1}^{\mu}
$$

$B$ makes

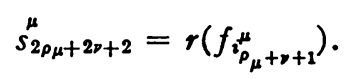

Otherwise let $\rho_{\mu+1},\left(0 \leqq \rho_{\mu+1} \leqq \rho_{\mu}+\nu\right)$ be the greatest integer satisfying for some $\alpha,(1 \leqq \alpha \leqq \mu)$

$$
r\left(f_{i_{\rho^{\prime}+\nu+1}^{\mu}}^{\mu}\right)-s_{2 \rho_{\mu+1}-1}^{\alpha}<k_{\rho_{\mu+1}} x_{2 \rho \mu+1-1}^{\alpha}
$$

(if $\rho_{\mu+1}=0$, take (2.2)) and $B$ turns to the $(\mu+1)$ st sequence of moves taking as $x_{2_{\rho_{\mu+1}}}^{\mu+1}$ the left side of (3.4) and making thus

$$
s_{2 \rho_{\mu+1}}^{\mu+1}=r\left(f_{i_{\mu}+\nu+1}^{\mu}\right) .
$$

Then $A$ has to make

$$
\underset{s_{2 \rho \mu+1+1}^{\mu+1}}{\mu+1} \in f_{i_{\rho+1}^{\mu+1}}^{\mu+1},\left(i_{\rho_{\mu+1}+1}^{\mu+1}>i_{\rho \mu+\nu+1}^{\mu}\right)
$$

and so on. If $A$ is to win, this procedure must come to an end after a finite number of moves with $s_{2 \rho_{\tau}+\delta+1}^{\tau} \in \in^{\prime} f_{m+1}$ for some integers $\tau, \delta$.

We shall now introduce a new notation to the moves described above. 


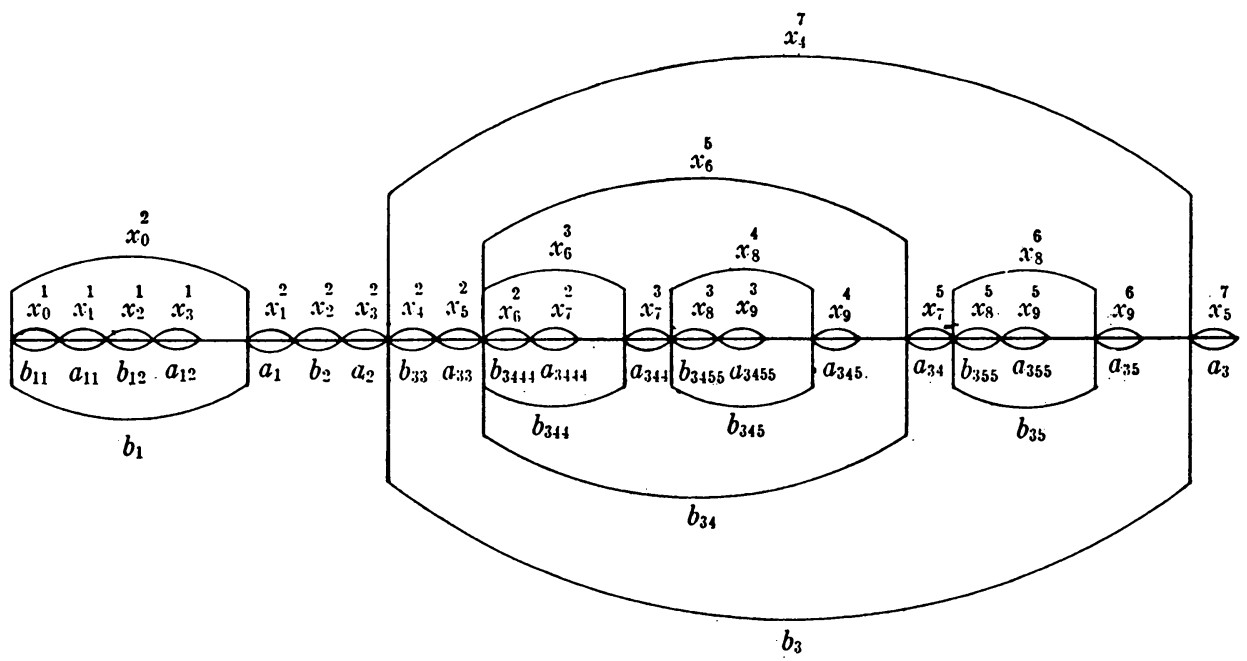

Looking at the move $x_{i}$ as the interval $\left[s_{i-1}, s_{i}\right],(i=0,1,2, \cdots)$ we shall denote

$$
b_{j_{1}}=x_{2 j_{1}-2}^{\alpha_{1}} \text { and } a_{j_{1}}=x_{2 j_{1}-1}^{\alpha_{1}}
$$

if $x_{2 j_{1}-2}^{\alpha_{1}}$ is not included in any other move. Further, $b_{j_{1}} \ldots j_{\gamma-1}$ being defined we denote

$$
b_{j_{1}} \cdots j_{\gamma-1} j_{\gamma}=\underset{x_{2 j_{\gamma-2}}^{\alpha \gamma}}{\alpha \gamma} b_{j_{1} \cdots j_{\gamma-1}} \text { and } a_{j_{1} \cdots j_{\gamma}}=\stackrel{\alpha_{2 \gamma}}{x_{2 j_{\gamma}-1}}
$$

if there exists no move $x_{2 v}^{\beta}$ satisfying

$$
x_{2 j_{\gamma-2}}^{\alpha_{\gamma}} \not \underset{x_{2 v}}{\beta} \neq b_{j_{1} \ldots j_{\gamma-1}} \neq x_{2 j_{\gamma-2}}^{\alpha_{\gamma}} \text { and } \quad x_{2 j_{\gamma}-2}^{\alpha_{\gamma}} \subset x_{2 \nu}^{\beta} \subset b_{j_{1} \ldots j_{\gamma-1}} \text {. }
$$

Evidently $j_{\gamma} \geqq j_{\gamma-1}$. The number of moves $x_{\nu}^{\mu}$ being finite so is the number of the intervals $a$ and $b$. Their numbers will be denoted:

$$
\begin{aligned}
b_{j_{1}} \cdots j_{\gamma}, a_{j_{1}} \cdots j_{\gamma},\left(j_{\lambda+1}\right. & =j_{\lambda}, j_{\lambda}+1, \cdots, t_{\lambda+1}=t_{\lambda+1}\left(j_{1}, j_{2}, \cdots, j_{\lambda}\right) ; \\
\lambda & \left.=0,1,2, \cdots, \gamma-1 ; \gamma=1,2, \cdots, h ; j_{0}=1\right) .
\end{aligned}
$$

The intervals $a_{j_{1}} \ldots j_{\gamma}$ are identical with the moves of $A$ and accordingly they cover $\mathrm{U}_{i=1}^{m} g_{i}$. Moreover those intervals are pairwise disjoint and therefore (3.2) and consequently also (3.1) will follow from

$$
\sum_{\gamma=1}^{h} \sum_{j_{\lambda-1} \leq j_{\lambda} \leq l_{\lambda} ; \lambda=1,2, \cdots, \gamma} a_{j_{1} \ldots j_{\gamma}}<1-\prod_{\mu=1}^{\infty} \eta_{\mu}
$$

which we proceed to prove.

From the construction of the intervals $a_{j}$ it is evident that 


$$
k_{j} a_{j} \leqq 1-\sum_{v=1}^{j} a_{\nu}-\sum_{v=1}^{j} b_{v}, \quad\left(j=1,2, \cdots, t_{1}\right) ;
$$

consequently, considering (3.3), we have

$$
a_{j} \leqq\left(1-\eta_{j}\right)\left(1-\sum_{v=1}^{j-1} a_{v}-\sum_{v=1}^{j} b_{v}\right), \quad\left(j=1,2, \cdots, t_{1}\right) .
$$

Using induction we shall verify the relation

$$
1-\sum_{\nu=1}^{p} a_{\nu}-\sum_{\nu=1}^{p} b_{\nu} \geqq \prod_{\nu=1}^{p} \eta_{\nu}-\sum_{\nu=1}^{p}\left(b_{\nu} \prod_{\mu=\nu}^{p} \eta_{\mu}\right), \quad\left(p=1,2, \cdots, t_{1}\right) .
$$

For $p=1$, (3.8) follows from (3.7) with $j=1$. Suppose now that (3.8) holds for some $p,\left(p<t_{1}\right)$. Putting in (3.7) $j=p+1$ we get

$$
\left(1-\sum_{\nu=1}^{p+1} a_{\nu}-\sum_{\nu=1}^{p+1} b_{\nu}\right)-\eta_{p+1}\left(1-\sum_{\nu=1}^{p} a_{\nu}-\sum_{\nu=1}^{p} b_{\nu}\right) \geqq-b_{p+1} \eta_{p+1}
$$

addition of inequality (3.8) multiplied by $\eta_{p+1}$ verifies (3.8) for $p+1$. Now put in (3.8) $p=t_{1}$. As the left side of (3.8) is positive we may-considering $0 \leqq \prod_{\mu=t_{1}+1}^{\infty} \eta_{\mu}<1$-multiply the right side of (3.8) with $\prod_{\mu=t_{1}+1}^{\infty} \eta_{\mu}$ and we get

$$
\sum_{\nu=1}^{t_{1}} a_{\nu}<\left(1-\prod_{\mu=1}^{\infty} \eta_{\mu}\right)-\sum_{\nu=1}^{t_{1}}\left[b_{\nu}\left(1-\prod_{\mu=\nu}^{\infty} \eta_{\mu}\right)\right]
$$

Similarly we have for every interval $b_{j_{1}} \cdots j_{\gamma-1}$

$$
\sum_{j_{\gamma}=j_{\gamma-1}}^{t_{\gamma}} a_{j_{1}} \cdots j_{\gamma}<b_{j_{1}} \cdots j_{\gamma-1}\left(1-\prod_{\mu=j_{\gamma}-1}^{\infty} \eta_{\mu}\right)-\sum_{j_{\gamma}=j_{\gamma-1}}^{t_{\gamma}}\left[b_{j_{1} \cdots j_{\gamma}}\left(1-\prod_{\mu=j_{\gamma}}^{\infty} \eta_{\mu}\right)\right]
$$

(for $\gamma=1$, take $b=[0,1]$ ). Summing up the inequalities (3.9) for all the intervals $b_{j_{1}} \ldots j_{\gamma-1}$, (see (3.5)) we get finally (3.6).

Theorem 2. Given a game $G\left\{k_{m}\right\}$, a necessary and sufficient condition for the existence of a perfect unavoidable set of measure 0 is

$$
\sum_{m=1}^{\infty} \frac{1}{k_{m}}=\infty
$$

Proof. In order to prove sufficiency we make use of the set $S$ constructed in the proof of Theorem 1. As shown there, $S$ is perfect and unavoidable. Moreover (2.1) gives

$$
m\left(S_{0}\right)=m(S \cap[0,1])=\prod_{i=1}^{\infty} 2 \frac{k_{i}+i}{2 k_{i}+2 i+1}=\prod_{i=1}^{\infty}\left(1-\frac{1}{2 k_{i}+2 i+1}\right)
$$

and from (3.10) follows $m\left(S_{0}\right)=0$. From (2.18) we get at last $m(S)=0$. 
For $\widetilde{G}\left\{k_{m}\right\}$ follows the necessity of (3.10) (for closed sets) from the lemma. We shall now extend the proof for $G\left\{k_{m}\right\}$. Let $\sum_{i=1}^{\infty} 1 / k_{i}<\infty$ and let $T$ be any closed unavoidable set $T \subset[0, \infty)$. If $m(T \cap[0,1])>0$, the theorem is proved; otherwise, $B$ is able-according to the lemma-to fix his strategy in such a way that either

$$
s \in C(T) \cap[0,1]
$$

or for some $n_{1}$

$$
s_{2 n_{1}}>1 \text {. }
$$

(3.11) contradicts the assumption that $T$ is unavoidable and thus (3.12) remains, i.e. $s_{2 n_{1}} \in\left[q_{1}, q_{1}+1\right)$ for some integer $q_{1}>0$. Generally let $s_{2 n_{i}} \in d_{i}$ $=\left[q_{i}, q_{i}+1\right)$. If $m\left(T \cap d_{i}\right)>0$ the theorem is proved. Otherwise $B$ can fix his strategy so that either $s \in C(T) \cap d_{i}$ which contradicts the unavoidableness of $T$ or for some $n_{i+1}>n_{i}, s_{2 n_{i+1}} \in d_{i+1}$ with $q_{i+1}>q_{i}$. This means however that $\lim _{i \rightarrow \infty} s_{2 n_{i}}=\lim _{j \rightarrow \infty} s_{j}=\infty$ which contradicts once more the assumption that $T$ is unavoidable.

REMARK 1. Theorem 2 remains true also with regard to the existence of a closed unavoidable set.

REMARK 2. The analogue of Remark 2 to Theorem 1 applies also to Theorem 2.

TheOREM 3. Given a game $\widetilde{G}\left\{k_{n}\right\}$, let $P\left\{k_{n}\right\}$ be the family of the perfect unavoidable subsets of $[0,1]$, then

$$
\inf _{S_{0} \in P\left\{k_{n}\right\}} m\left(S_{0}\right)=\prod_{i=1}^{\infty}\left(1-\frac{1}{k_{i}+1}\right) .
$$

REMARK. The theorem remains true also for closed unavoidable sets. In order to prove both propositions it must be shown that the measure of every closed unavoidable subset of $[0,1]$ is not less then $\prod_{i=1}^{\infty} \eta_{i}$ (see (3.3)) which follows from the lemma, and that for every $\epsilon>0$ there exists a set $S_{0} \in P\left\{k_{n}\right\}$ such that

$$
m\left(S_{0}\right) \leqq \prod_{i=1}^{\infty} \eta_{i}+\epsilon,
$$

which will now be proved.

Proof. In the case that (3.10) holds; our proposition follows from Theorem 2. It remains therefore to consider

$$
\sum_{i=1}^{\infty} \frac{1}{k_{i}}<\infty
$$

As in proof of Theorem 1 we shall construct a perfect set $S_{0}$ satisfying (3.13), which will turn out to be unavoidable. 
Let $f=[0,1]$ and decompose $f$ into 3 subintervals

$$
f_{0}=\left[0, \frac{1}{k_{1}+2}\right], \quad g=\left(\frac{1}{k_{1}+2}, \frac{2}{k_{1}+2}\right), \quad f_{1}=\left[\frac{2}{k_{1}+2}, 1\right] .
$$

Similarly decompose for every $n,(n=1,2, \cdots)$ each of the closed intervals $f_{\delta_{1}} \cdots \delta_{n-1},\left(\delta_{i}=0,1 ; i=1,2, \cdots, n-1\right)$

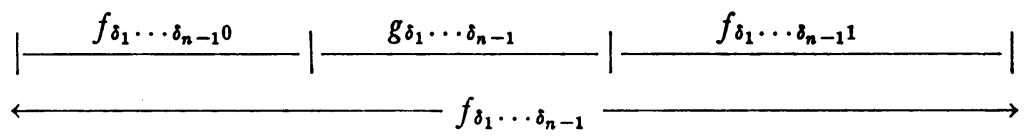

into 2 closed subintervals $f_{\delta_{1}} \cdots \delta_{n-1} \delta_{n},\left(\delta_{n}=0,1\right)$ and an open one $g_{\delta_{1}} \cdots \delta_{n-1}$ so that

$$
\left|f_{\delta_{1}} \ldots \delta_{n-1} 0\right|=\left|g_{\delta_{1}} \ldots \delta_{n-1}\right|=\frac{1}{k_{\gamma_{n}}+2}\left|f_{\delta_{1} \cdots \delta_{n-1}}\right|
$$

$$
\left|f_{\delta_{1}} \ldots \delta_{n-1}\right|=\frac{k_{\gamma_{n}}}{k_{\gamma_{n}}+2}\left|f_{\delta_{1}} \ldots \delta_{n-1}\right|
$$

where

$$
\gamma_{n}=1+\sum_{i=1}^{n-1} \delta_{i}
$$

We denote

$$
S^{\prime}=\bigcap_{n=0}^{\infty} \underset{\delta_{i}=0,1 ; i=1,2, \cdots, n}{U} f_{\delta_{1}} \ldots \delta_{n} .
$$

Further let $\epsilon>0$ be any positive number. We form the closed intervals

$$
\begin{array}{r}
d_{\delta_{1} \ldots \delta_{n}}=\left[r\left(g_{\delta_{1}} \ldots \delta_{n}\right)-\frac{\epsilon}{2^{2 n+2}}\left|g_{\delta_{1}} \ldots \delta_{n}\right|, r\left(g_{\delta_{1}} \cdots \delta_{n}\right)+\frac{\epsilon}{2^{2 n+2}}\left|g_{\delta_{1}} \cdots \delta_{n}\right|\right], \\
\left(\delta_{i}=0,1 ; i=1,2, \cdots, n ; n=0,1,2, \cdots\right)
\end{array}
$$

and put

$$
D=\bigcup_{n=0}^{\infty} \underset{\delta_{i}=0,1 ; i=1,2, \ldots, n}{\bigcup} d_{\delta_{1} \ldots \delta_{n}}
$$

Let now

$$
S_{0}=\left(S^{\prime} \cup D\right) \cap[0,1] .
$$

Evidently $S^{\prime}$ is perfect. It follows immediately that $S_{0}$ is dense in itself. In order to prove that $S_{0}$ is also closed, the only nontrivial case arises when $z=\lim _{n \rightarrow \infty} z_{\delta_{1}} \ldots \delta_{n}$ for some sequence $\delta_{1}, \delta_{2}, \cdots\left(\delta_{i}=0,1 ; i=1,2, \cdots\right)$ where 
$z_{\delta_{1}} \ldots \delta_{n} \in d_{\delta_{1} \ldots \delta_{n}}$. It must be proved that $z \in S_{0}$. This follows however from (3.17) as $z=\lim _{n \rightarrow \infty} z_{\delta_{1}} \cdots \delta_{n}=\lim _{n \rightarrow \infty} r\left(g_{\delta_{1}} \cdots \delta_{n}\right) \in S^{\prime} \subset S_{0}$. Consequently $S_{0}$ is perfect.

We shall now show that (3.13) is satisfied. $m\left(S^{\prime}\right)$ is some function of the sequence $k_{i},(i=1,2, \cdots)$, say $m\left(S^{\prime}\right)=\psi\left(k_{1}, k_{2}, \cdots\right)$. The method of construction of $S^{\prime}$ shows that $m\left(S^{\prime} \cap f_{0}\right)=\left(1 /\left(k_{1}+2\right)\right) \psi\left(k_{1}, k_{2}, \cdots\right)$ and $m\left(S^{\prime} \cap f_{1}\right)$ $=\left(k_{1} /\left(k_{1}+2\right)\right) \psi\left(k_{2}, k_{3}, \cdots\right)$, i.e. $\psi\left(k_{1}, k_{2}, \cdots\right)=\left(1 /\left(k_{1}+2\right)\right) \psi\left(k_{1}, k_{2}, \cdots\right)$ $+\left(k_{1} /\left(k_{1}+2\right)\right) \psi\left(k_{2}, k_{3}, \cdots\right)$. Considering (3.3) we have

$$
\psi\left(k_{1}, k_{2}, \cdots\right)=\eta_{1} \psi\left(k_{2}, k_{3}, \cdots\right) .
$$

Similarly we have in the general case for $n=1,2, \ldots$

$$
m\left(S^{\prime} \cap f_{\delta_{1}} \ldots \delta_{j}\right)=\left|f_{\delta_{1}} \ldots \delta_{j}\right| \psi\left(k_{n}, k_{n+1}, \cdots\right),
$$

where $\delta_{1}, \cdots, \delta_{j}$ is any sequence of 0 's and 1 's satisfying $\sum_{i=1}^{j} \delta_{i}=n-1$. Now

$$
m\left(S^{\prime} \cap f_{\delta_{1}} \ldots \delta_{j}\right)=m\left(S^{\prime} \cap f_{\delta_{1}} \ldots \delta_{j} 0\right)+m\left(S^{\prime} \cap f_{\delta_{1}} \ldots \delta_{j} 1\right)
$$

and considering (3.15) we have

$$
\begin{aligned}
& \left|f_{\delta_{1}} \ldots \delta_{j}\right| \psi\left(k_{n}, k_{n+1}, \cdots\right) \\
& =\left|f_{\delta_{1}} \ldots \delta_{j}\right|\left\{\frac{1}{k_{n}+2} \psi\left(k_{n}, k_{n+1}, \cdots\right)+\frac{k_{n}}{k_{n}+2} \psi\left(k_{n+1}, k_{n+2}, \cdots\right)\right\},
\end{aligned}
$$

and in the same way as (3.19) we obtain

$$
\psi\left(k_{n}, k_{n+1}, \cdots\right)=\eta_{n} \psi\left(k_{n+1}, k_{n+2}, \cdots\right), \quad(n=1,2, \cdots) .
$$

It follows immediately

$$
\psi\left(k_{1}, k_{2}, \cdots\right)=\prod_{i=1}^{n} \eta_{i} \cdot \psi\left(k_{n+1}, k_{n+2}, \cdots\right), \quad(n=1,2, \cdots) .
$$

Now evidently $\lim \sup _{n \rightarrow \infty} \psi\left(k_{n+1}, k_{n+2}, \cdots\right) \leqq 1$ and consequently $m\left(S^{\prime}\right)$ $=\psi\left(k_{1}, k_{2}, \cdots\right) \leqq \prod_{i=1}^{\infty} \eta_{i}$. Moreover from (3.17) we have that $m(D)<\epsilon$, and therefore from (3.18) follows (3.13).

It remains to be proved that $A$ has a winning strategy on $S_{0}$. In the first place we remark that $B$ should avoid

$$
\begin{aligned}
s_{2 n} \in{ }^{\prime} d_{\rho_{1} \cdots \rho_{j}}, \quad\left(\rho_{i}=0,1 ; i=1,2, \cdots, j ; j=0,1,2, \cdots ;\right. \\
n=0,1,2, \cdots),
\end{aligned}
$$

as in that case $A$ would evidently win just by choosing $x_{2 i+1},(i=n, n+1, \cdots)$ small enough.

$A$ will be said to be in a winning position after his $n$th move, $A \in W_{n}^{\prime \prime}$, if for some $d_{\delta_{1} \cdots \delta_{m-1}},\left(\gamma_{m} \geqq n\right)$ 
$\left(\alpha_{n}{ }^{\prime \prime}\right)$

$$
s_{2 n-1}=l\left(d_{\delta_{1} \cdots \delta_{m-1}}\right)
$$

and

$\left(\beta_{n}{ }^{\prime \prime}\right)$

$$
k_{n} x_{2 n-1} \leqq\left|f_{\delta_{1}} \ldots \delta_{m-1}\right| .
$$

We shall prove that if (3.20) never occurs, then $A$ has a strategy which enables him to be in a winning position after each of his moves. From this follows $s_{2 n-1} \in S_{0},(n=1,2, \cdots)$ and $-S_{0}$ being closed and bounded-also $\lim _{n \rightarrow \infty} s_{2 n-1}=s \in S_{0}$, i.e. $S_{0}$ is unavoidable.

Agreeing on (2.2), $A \in W_{0}^{\prime \prime}$. Suppose now that $A \in W_{n}^{\prime \prime}$. Considering (3.20), $B$ has to make

$$
s_{2 n} \in f_{\delta_{1} \ldots \delta_{m-1} \delta_{m}}^{\circ}
$$$$
\left(\delta_{m}=1\right) .
$$

If for some

$$
g_{\delta_{1}} \ldots \delta_{\lambda},(\lambda \geqq m), s_{2 n} \in^{\prime}\left(g_{\delta_{1}} \ldots \delta_{\lambda} \cap C\left(d_{\delta_{1}} \ldots \delta_{\lambda}\right)\right),
$$

then $A$ makes

$$
s_{2 n+1}=l\left(d_{\delta_{1}} \ldots \delta_{\lambda}\right)
$$

Of course

$$
x_{2 n+1}<\left|g_{\delta_{1} \ldots \delta_{\lambda}}\right|
$$

and considering (3.15) and $\left(\alpha_{n}^{\prime \prime}\right)$ this move satisfies (1.1). Moreover from (3.21) follows $\gamma_{\lambda+1} \geqq \gamma_{m}+1 \geqq n+1$, and therefore (3.22) gives $\left(\alpha_{n+1}^{\prime \prime}\right)$; at last (3.15) and (3.23) give

$$
k_{n+1} x_{2 n+1} \leqq k_{\gamma_{\lambda+1}} x_{2 n+1}<\left|f_{\delta_{1}} \ldots \delta_{\lambda 1}\right|
$$

which confirms $\left(\beta_{n+1}^{\prime \prime}\right)$. Consequently $A \in W_{n+1}^{\prime \prime}$.

As (3.20) should be avoided the only other possibility is that (2.4) holds, and there are infinitely many zeros-and considering (3.20)-also infinitely many 1's among the indices of $f_{\delta_{1} \delta_{2}} \ldots$. Accordingly by (3.16), $\gamma_{i} \rightarrow \infty$. Moreover (3.14) implies $k_{i} \rightarrow \infty$, and consequently there exists an integer $\mu \geqq m+1$ such that

$$
\begin{aligned}
k_{\gamma_{\mu}} & \geqq 2 k_{n+1}, \\
2\left|g_{\delta_{1}} \ldots \delta_{\mu-1}\right| & <x_{2 n}
\end{aligned}
$$

and $\delta_{\mu}=0$, i.e.

$$
s_{2 n} \in f_{\delta_{1}}^{\circ} \cdots \delta_{\mu-1} 0 .
$$

Now $A$ makes $s_{2 n+1}=l\left(d_{\delta_{1}} \ldots \delta_{\mu-1}\right)$. By (3.15), (3.26) and (3.25) this move satisfies (1.1). Further considering the selfevident $\gamma_{\mu} \geqq \gamma_{m}+1 \geqq n+1,\left(\alpha_{n+1}^{\prime \prime}\right)$ is fulfilled, and by (3.24), (3.26) and (3.15) 


$$
k_{n+1} x_{2 n+1} \leqq \frac{1}{2} k_{\gamma \mu} \cdot 2\left|g_{\delta_{1} \cdots \delta_{\mu-1}}\right|=\left|f_{\delta_{1} \cdots \delta_{\mu-1} 1}\right|,
$$

i.e. $\left(\beta_{n+1}^{\prime \prime}\right)$ is satisfied as well. Consequently $A \in W_{n+1}^{\prime \prime}$.

4. On universal unavoidable sets. In a natural way there arises the problem of describing the structure of a universal unavoidable set UUSG i.e. a set which is unavoidable for every game $G\left\{k_{m}\right\}$ and-respectively-of a UUS $\tilde{G}$ i.e. a subset of $[0,1]$ which is unavoidable for every game $\tilde{G}\left\{k_{m}\right\}$.

For closed sets it follows from the lemma that if $S_{0}$ is a UUSG then $m\left(S_{0}\right)=1$, i.e. $S_{0}=[0,1]$. More generally we shall prove

THEOREM 4. A UUSG is of the second category at every point of $[0,1]$.

Proof ( $\left.{ }^{1}\right)$. Suppose that $S_{0} \subset[0,1]$ is of the first category at some point of $[0,1]$. We shall show that $S_{0}$ is not a $U U S \tilde{G}$ by constructing a sequence

$$
0<k_{1}^{*} \leqq k_{2}^{*} \leqq \cdots
$$

such that for $\widetilde{G}\left\{k_{m}^{*}\right\}, B$ has a winning strategy on $S_{0}$.

Let $d$ be a closed interval contained in $(0,1)$ such that $S_{0} \cap d$ is of first category. Let $G_{n},(n=1,2, \cdots)$ be a sequence of open sets, each dense in $d$, such that their intersection $E=\bigcap_{n-1}^{\infty} G_{n}$ is contained in $d \cap C\left(S_{0}\right)$. Let further $R$ denote the set of all rational numbers $r$ in the interval $[0,|d| / 2]$, and define $E_{0}=\bigcap_{r \in R}(E-r) . E_{0}$ is not empty, since it includes the first half of $d$, except for a set of first category.

Choose arbitrarily a point $x_{0} \in E_{0}$ and a real number $M \geqq 2$. Let $N_{0}$ be an integer such that

$$
2^{N_{0}} \geqq \max (M, 2 /|d|) .
$$

Note that $x_{0}+r \in E$ for every rational number $r$ in the interval $\left[0,2^{-N_{0}}\right]$. Let $T_{0}=\left\{x_{0}\right\}$ and for each $n \geqq 1$ let

$$
T_{n}=\left\{x_{0}+2^{-i_{1}}+2^{-i_{2}}+\cdots+2^{-i_{n}}: N_{0}<i_{1}<i_{2}<\cdots<i_{n}\right\}
$$

where $i_{k}$ denote positive integers. ( $T_{n}$ consists of all numbers $x_{0}+r$, where $r$ is a dyadic rational less than $2^{-N_{0}}$ having exactly $n$ binary digits different from zero.) Then

$$
\bar{T}_{n}=\bigcup_{k=0}^{n} T_{k} .
$$

Hence $\bar{T}_{n}$ is a compact set and $\bar{T}_{n} \subset E$ for every $n$. Therefore $\bar{T}_{n-1} \subset G_{n}$, $(n=1,2, \cdots)$, and since $G_{n}$ is open there exists an increasing sequence of integers

(1) The author is indebted to Professor J. C. Oxtoby for this proof, which is much shorter than the original one. 


$$
N_{0}<N_{1}<N_{2}<\cdots
$$

such that

$$
F_{n}=\bigcup_{x \in \bar{T}_{n-1}}\left[x, x+2^{-N_{n}+1}\right] \subset G_{n}, \quad(n=1,2, \cdots) .
$$

The sets $F_{n}$ are compact and so is their intersection $F=\bigcap_{n=1}^{\infty} F_{n}$ which is evidently contained in $E$. Put

$$
D_{n}=\left\{x_{0}+2^{-i_{1}}+2^{-i_{2}}+\cdots+2^{-j_{n}} ; j_{k} \geqq N_{k}\right\}, \quad(n=0,1,2, \cdots)
$$

and note that

$$
D_{n} \subset F \subset E, \quad(n=0,1,2, \cdots) \text {. }
$$

We define now the sequence (4.1) putting

$$
k_{m}^{*}=2^{N_{m}+N_{0}}
$$

and show that for the game $\tilde{G}\left\{k_{m}^{*}\right\}, B$ has a winning strategy on $S_{0}$.

$B$ is said to be in a winning position after his $(n+1)$-st move $x_{2 n}, B \in W_{n}$, if $s_{2 n} \in D_{n}$.

Choosing $x_{0}$ as above, $B \in W_{0}$. Suppose now that $B \in W_{n}$. Considering $D_{n} \subset[0,1]$ and $(1.1)$, however $A$ chooses $x_{2 n+1}$, there will hold $s_{2 n+1}<M$. Now if $A$ chooses $x_{2 n+1} \geqq 2^{-N_{n+1}}, B$ makes $s_{2 n+2}=M$, which by (4.2) and (4.4) satisfies (1.2), and wins. If, however, $A$ chooses $x_{2 n+1}<2^{-N_{n+1}}$, let $j_{n+1}$ be the largest integer satisfying $x_{2 n+1}<2^{-j_{n+1}}$. $B$ chooses then $x_{2 n+2}=2^{-j_{n+1}}-x_{2 n+1}$, which evidently satisfies (1.2) as in this case $x_{2 n+2}<x_{2 n+1}$. Thus $s_{2 n+2} \in D_{n+1}$ and $B \in W_{n+1}$.

We have thus shown that-being in a winning position- $B$ can always manage either to win immediately or to reach another winning position. If, however, $B \in W_{n},(n=0,1,2, \cdots)$, i.e. $s_{2 n} \in D_{n}, s=\lim _{n \rightarrow \infty} s_{2 n}$ is included in the closure of $\cup_{n=1}^{\infty} D_{n}$ and by (4.3), $s \in F \subset E \subset d \cap C\left(S_{0}\right)$ and $B$ wins again.

With respect to the sets $U U S G$ we shall prove

ThEOREM 5. A UUSG is (for some $M \geqq 0$ ) of the second category in every point of $[M, \infty)$.

Proof. Suppose there is a set $S$ which does not satisfy the above condition. It follows that there exists a sequence $d^{0}, d^{1}, d^{2}, \cdots$ of closed intervals such that

$$
l\left(d^{q+1}\right)>2 r\left(d^{q}\right), \quad(q=0,1,2, \cdots)
$$

and that $S \cap d^{q}$ is of the first category for each $q$. We shall prove that $S$ is not a UUSG; we shall namely construct a sequence

$$
0<\breve{k}_{1} \leqq \breve{k}_{2} \leqq \cdots
$$


such that for $G\left\{\widetilde{k}_{m}\right\}, B$ will have a winning strategy on $S$.

To this end denote

$$
M^{q}=r\left(d^{q+1}\right)-l\left(d^{q}\right)
$$

and construct for every $d^{q},(q=0,1,2, \cdots)$ with regard to $S \cap d^{q}$ and $M^{q}$, a point $x_{0}^{q}$, sets $D_{n}^{q},(n=0,1,2, \cdots)$ and the sequences:

$$
\begin{aligned}
N_{0}^{q} & <N_{1}^{q}<N_{2}^{q}<\cdots, \\
0 & <k_{1}^{q}<k_{2}^{q}<\cdots,
\end{aligned}
$$

in the same way as the point $x_{0}$, the sets $D_{n}$ and the sequences $\left\{N_{n}\right\}$ and $\left\{k_{m}^{*}\right\}$ have been constructed for $d$ with regard to $S_{0} \cap d$ and $M$ in the proof of Theorem 4. Further define the monotone sequence (4.6) putting

$$
\widetilde{k}_{n}=\max _{0 \leqq q \leqq n-1} k_{n}^{q} .
$$

The strategy of $B$ will now be defined. $B$ is said to be in a winning position after his $(n+1)$ st move $x_{2 n}, B \in W_{n}^{\prime}$, if $s_{2 n} \in D_{m}^{q}$ for some $q, 0 \leqq q \leqq n$ and some $m, 0 \leqq m \leqq n$.

Choosing as the first move $x_{0}=x_{0}^{0} \in D_{0}^{0}, B \in W_{0}^{\prime}$. Suppose now that $B \in W_{n}^{\prime}$, i.e. $s_{2 n} \in D_{m}^{q},(0 \leqq q \leqq n, 0 \leqq m \leqq n)$. If $A$ chooses

$$
x_{2 n+1}<2^{-N_{m+1}^{q}}
$$

let $j_{m+1}^{a}$ be the largest integer satisfying $x_{2 n+1}<2^{-i_{m+1}}$. $B$ chooses then $x_{2 n+2}$ $=2^{-j_{m+1}^{q}}-x_{2 n+1}$ and thus $s_{2 n+2} \in D_{m+1}^{q}$ or $B \in W_{n+1}^{\prime}$. If however $A$ chooses

$$
x_{2 n+1} \geqq 2^{-N_{m+1}^{q}}
$$

we have anyhow by (1.1) and (4.5), $s_{2 n+1}<l\left(d^{q+1}\right)<x_{0}^{q+1}$. $B$ makes then $s_{2 n+2}=x_{0}^{q+1} \in D_{0}^{q+1}$ which considering $x_{0}^{q+1}-s_{2 n+1}<x_{0}^{q+1}-l\left(d^{q}\right)<M^{q}$ $\leqq 2^{N_{0}^{q}} \leqq \breve{k}_{n+1} x_{2 n+1}$ satisfies (1.2), and $B \in W_{n+1}^{\prime}$.

Now if for every sufficiently large $n, A$ chooses for $x_{2 n+1}$ moves $(\alpha)$ only, then there exist $N$ and $q$ such that $s_{2 n} \in d^{q},(n \geqq N)$ and $B$ has a winning strategy on $d^{q}$. Otherwise $\lim _{n \rightarrow \infty} s_{2 n}=\infty$ and $B$ wins again.

A sufficient condition for a set $S \subset[0, \infty)$ to be a $U U S G$ will now be given. The condition itself and the proof of its sufficiency are only a slight generalization of the respective problem for the game $G\left\{k_{m}\right\},\left(k_{1}=k_{2}=\cdots=1\right)$ solved by $M$. Reichbach [3] (see also [2]). Further we shall construct a UUSG whose complement $C(S)$ is a countable union of perfect sets and is dense in $[0, \infty)$.

We begin with the definition of the property $\mathrm{H}$.

$\mathrm{H}$ : A set $P \subset[0, \infty)$ is said to have the property $\mathrm{H}$ if, for every $x \geqq 0$ and every two numbers $\epsilon>0$ and $k>0$, there exists to the right of $x$ an interval $g$ such that $g \cap P=\varnothing$ and $k(l(g)-x) \leqq|g|<\epsilon$. 
For every game $G\left\{k_{m}\right\}, A$ has a winning strategy on $C(P)$ and by an analogous method as used in the proof of Theorem 2 [3] we obtain

Theorem 6. Let $N=\bigcup_{i=0}^{\infty} N_{i}$ be a union of sets $N_{i}$ having the property $\mathrm{H}$, then $C(N)$ is a UUSG.

Note that every finite set has the property $\mathrm{H}$ and therefore the complement of every countable set is a UUSG.

We give now an example of a perfect set $N_{0}$ having the property $\mathrm{H}$. Let $f=[0,1]$, and decompose $f$ into 3 subintervals

$$
f_{0}=\left[0, \frac{1}{3}\right], \quad g=\left(\frac{1}{3}, \frac{2}{3}\right), \quad f_{1}=\left[\frac{2}{3}, 1\right] .
$$

Similarly decompose, for every $n,(n=1,2, \cdots)$, each of the closed intervals $f_{\delta_{1}} \ldots \delta_{n-1},\left(\delta_{i}=0,1 ; i=1,2, \cdots, n-1\right)$ into 2 closed

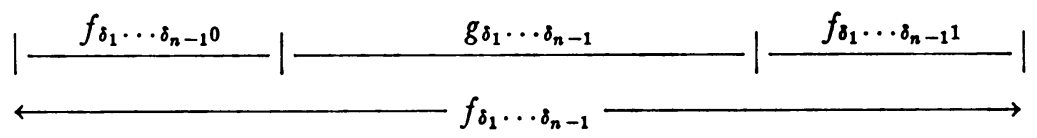

subintervals $f_{\delta_{1}} \cdots \delta_{n-1} \delta_{n},\left(\delta_{n}=0,1\right)$ and an open one $g_{\delta_{1}} \cdots \delta_{n-1}$ so that

$$
\left|f_{\delta_{1}} \ldots \delta_{n}\right|=\frac{1}{n+2}\left|f_{\delta_{1} \cdots \delta_{n-1}}\right|,\left(\delta_{n}=0,1\right) ;\left|g_{\delta_{1} \cdots \delta_{n-1}}\right|=\frac{n}{n+2}\left|f_{\delta_{1} \cdots \delta_{n-1}}\right| \text {. }
$$

The set

$$
N_{0}=\bigcap_{n=0}^{\infty} \sin _{i=0,1 ; i=1,2, \cdots, n} f_{\delta_{1}} \ldots \delta_{n}
$$

is perfect and has evidently the property $H$.

Now denoting by $\left\{r_{i}\right\}$ the sequence of all positive rational numbers and putting $N_{i}=N_{0}+r_{i}$ we obtain from Theorem 6 that $S=C\left(\bigcup_{i=0}^{\infty} N_{i}\right)$ is a UUSG. Similarly it can be seen that $S \cap[0,1]$ is a $U U S \tilde{G}$.

\section{BIBLIOGRAPHY}

1. H. Hanani and M. Reichbach, Some characterizations of unavoidable compact sets in the game of Banach and Mazur, to appear.

2. S. Hartman, Bemerkungen über das Spiel von Banach und Mazur, Colloq. Math. vol. 4 (1957) p. 261.

3. M. Reichbach, Ein Spiel von Banach und Mazur, Colloq. Math. vol. 5 (1957) pp. 16-23.

4. A. Turowicz, Sur une proprieté des nombres irrationels, Ann. Polon. Math. vol. 2 (1955) pp. 103-105.

5. S. Zubrzycki, On the game of Banach and Mazur, Colloq. Math. vol. 4 (1957) pp. 227-229.

ISRAEL INSTITUTE OF TEChNOLOGY,

HAIFA, ISRAEL 